\title{
Expectations and the Stability Problem for Optimal Monetary Policies*
}

\author{
George W. Evans ${ }^{\dagger}$ \\ University of Oregon
}

\author{
Seppo Honkapohja \\ University of Helsinki
}

July 3, 2001: revised

\begin{abstract}
A fundamentals based monetary policy rule, which would be the optimal monetary policy without commitment when private agents have perfectly rational expectations, is unstable if in fact these agents follow standard adaptive learning rules. This problem can be overcome if private expectations are observed and suitably incorporated into the policy maker's optimal rule. These strong results extend to the case in which there is simultaneous learning by the policy maker and the private agents. Our findings show the importance of conditioning policy appropriately, not just on fundamentals, but also directly on observed household and firm expectations.
\end{abstract}

JEL classifications: E52, C62, D83, D84, C62

*An earlier version of this paper was presented at the Deutsche Bundesbank Conference on Transparency in Monetary Policy, the Federal Reserve Bank of Cleveland Workshop on Learning and Model Misspecification, the NBER Monetary Economics Program meeting, the 2001 SED Congress and in numerous seminars. We thank the audiences for useful comments. We would especially like to thank (without implicating) Gabriel Desgranges, Van Kolpin, Ben McCallum, Kaushik Mitra, Athanasios Orphanides, Christopher Sims, Michael Woodford and the referees for their comments. Financial support from the US National Science Foundation, Academy of Finland, Yrjö Jahnsson Foundation and Nokia Group is gratefully acknowledged.

${ }^{\dagger}$ Address of corresponding author: Professor George W. Evans, Department of Economics, 1285 University of Oregon, Eugene, OR 97403-1285, USA. email:gevans@oregon.uoregon.edu. 


\section{Introduction}

The formulation and performance of monetary policy rules has been analyzed extensively in the recent literature, for recent surveys see e.g. (Clarida, Gali, and Gertler 1999), (Woodford 1999) and (McCallum 1999). Much of the recent analysis has been conducted using the "New Phillips curve" model, also known as the optimizing IS-AS model, derived in a number of papers and reviewed e.g. in (Clarida, Gali, and Gertler 1999) and (Woodford 1999). This model gives a central role for private sector expectations of inflation and future output.

The corresponding monetary policy literature includes both the study of optimal policy, for specified objective functions, and analysis of the performance of simple, not necessarily optimal rules, such as Taylor's interest rate feedback rule (Taylor 1993). Examination of optimal policy in turn can be divided into the study of "time consistent" policy, under discretion, and policy in which the monetary authorities can commit to a rule that constrains future policy. As is well known, under rational expectations there can be gains, as measured by the policy maker's objective function, when a binding commitment to a rule is possible. However, it may not be considered credible for policy makers to commit to a rule from which there will be future incentives to deviate. Optimal rules without commitment have the property that the monetary authorities have no incentive to change policy even though they have the discretion to do so.

While almost all of the literature has assessed the design of monetary policy rules under rational expectations (RE), this may not be an innocuous assumption. One major difficulty is that some policy rules can yield indeterminacy of equilibria, i.e. multiple RE solutions. This has been demonstrated in (Bernanke and Woodford 1997), (Woodford 1999) and (Svensson and Woodford 1999).

A second major problem is that these rules may not perform satisfactorily if instead of having RE private agents follow plausible learning rules. Recently, (Bullard and Mitra 2000) have shown that if agents follow adaptive learning rules, then the stability of the Taylor-type rules could not be taken for granted. An earlier paper by (Howitt 1992) showed the instability under learning of interest rate pegging and related rules in both flexible price and IS-LM type models. In his conclusions Howitt explicitly warned that any analysis of monetary policy under RE should be supplemented with an investigation of its stability under learning. 
We take up this issue in the context of optimal policy rules without commitment, and obtain some strong results. ${ }^{1}$ We begin by showing that the economy is unstable if the policy makers follow the optimal interest rate rule formulated in terms of observed fundamental shocks and private agents follow natural learning rules to form expectations of inflation and future output. Small expectational errors by private agents become magnified by the policy, which assumes RE, and the cumulative process drives the economy away from the rational expectations equilibrium (REE). This result can be viewed as an extension of Howitt's results to the New Phillips curve framework.

A central objective of the paper is to show how this problem can be overcome. In particular, we seek a monetary policy which both is stable under learning and implements optimal discretionary policy. The key to achieving a stable optimal monetary policy is to formulate a rule that explicitly takes account of private sector expectations and the economic structure. We obtain a striking result. We show that under a suitably designed rule of this type, the economy will invariably be stable under learning and will converge over time to the REE corresponding to the optimal policy without commitment. Furthermore, we show that such rules continue to be stable under learning even if the policy maker also has to learn the true structural parameters required to conduct optimal monetary policy under discretion.

In practice, monetary authorities appear to base their policy on a variety of indicators that include consumer and business sentiments. For example, Bank of England Inflation Reports, see (Bank of England 2001), directly discuss private sector forecasts, while (Blinder 1998), pp. 60-61, poses the question of whether Central Bankers should "follow the markets," which "sometimes stray far from fundamentals." 2 From the strict RE viewpoint such practices might seem puzzling since with a unique REE these expectations are functions of the observable fundamentals. Possible ways to explain the practices of the policy maker include multiplicities of REE and informational asymmetries. This paper provides a different explanation, which does not hinge on these factors. The possibility of small deviations from RE leading to destabilizing fluctuations is enough to justify careful attention to the expectations of private agents.

\footnotetext{
${ }^{1}$ Policy with a limited form of commitment possible is discussed in Section 4.3.2. We leave for future research a general study of learning stability for optimal monetary policy rules when full commitment is possible.

${ }^{2}$ (Hall 1984), p.146, explicitly proposed that the "Fed's internal procedure" should place some weight on "reliable outside forecasts."
} 


\section{The Key Results}

\subsection{The Basic Model}

We utilize the standard log-linear framework as developed in Section 2 of (Clarida, Gali, and Gertler 1999). The structural model consists of two equations:

$$
\begin{gathered}
x_{t}=-\varphi\left(i_{t}-\hat{E}_{t} \pi_{t+1}\right)+\hat{E}_{t} x_{t+1}+g_{t} \\
\pi_{t}=\lambda x_{t}+\beta \hat{E}_{t} \pi_{t+1}+u_{t},
\end{gathered}
$$

where $x_{t}$ is the "output gap" i.e. the difference between actual and potential output, $\pi_{t}$ is the inflation rate, i.e. the proportional rate of change in the price level from $t-1$ to $t$ and $i_{t}$ is the nominal interest rate. All the parameters in (1) and (2) are positive. $0<\beta<1$ is the discount rate of the representative firm and is therefore close to one. $\hat{E}_{t} x_{t+1}$ and $\hat{E}_{t} \pi_{t+1}$ denote private sector expectations of inflation and output gap next period. These expectations may or may not be rational and we interpret this model as giving the temporary equilibrium of the economy, i.e. the period $t$ equilibrium for given expectations and exogenous shocks. We will use the same notation without the "^" to denote RE. In this section we assume RE. In Section 2.2 we discuss the interpretation of a temporary equilibrium outside an REE.

(1) is a dynamic "IS" curve which can be derived from the Euler equation associated with the household's savings decision. (2) is a "new Phillips" curve which can be derived from optimal pricing decisions of monopolistically competitive firms facing constraints on the frequency of future price changes. The essence of the new Phillips curve is the forward-looking character of the inflation expectations. Finally, $g_{t}$ and $u_{t}$ denote observable shocks following first order autoregressive processes

$$
\begin{gathered}
g_{t}=\mu g_{t-1}+\tilde{g}_{t} \\
u_{t}=\rho u_{t-1}+\tilde{u}_{t},
\end{gathered}
$$

where $0<|\mu|<1,0<|\rho|<1$ and $\tilde{g}_{t} \sim \operatorname{iid}\left(0, \sigma_{g}^{2}\right), \tilde{u}_{t} \sim \operatorname{iid}\left(0, \sigma_{u}^{2}\right) . \quad g_{t}$ represents shocks to government purchases as well as shocks to potential 
GDP. $u_{t}$ represents any cost push shocks to marginal costs other than those entering through $x_{t}$.

It should be emphasized that the model (1) and (2) is derived by suitably linearizing a nonlinear framework around a nonstochastic steady state. Any analysis based on linearization must be interpreted as being local in a neighborhood of the steady state. In particular, the random shocks must be sufficiently small for the linearization to make sense. We also follow the common practice of leaving hidden the government budget constraint and the equation for the evolution of government debt. This is acceptable provided fiscal policy appropriately accommodates the consequences of monetary policy for the government budget constraint. ${ }^{3}$

We now take up the standard formulation of optimal discretionary policy under rational expectations, following (Clarida, Gali, and Gertler 1999). The policy maker has a standard objective function defined in terms of the target variables $x_{t}$ and $\pi_{t}$ :

$$
\min \frac{1}{2} E_{t}\left\{\sum_{i=0}^{\infty} \beta^{i}\left[\alpha\left(x_{t+i}-\bar{x}\right)^{2}+\left(\pi_{t+i}-\bar{\pi}\right)^{2}\right]\right\},
$$

where $\bar{x}$ allows for a possible deviation of socially optimal output from potential output and $\bar{\pi}$ is the target value for the inflation rate. (If desired, these can be assumed to be zero.) $\alpha$ is the relative weight for output deviations, and $\beta$ is the discount rate. Thus the policy maker discounts the future at the same rate as the private sector. We note that $\alpha=0$ would correspond to pure inflation targeting.

Optimal policy without commitment reduces to a sequence of static problems in which the nominal interest rate is chosen to deliver the values $x_{t}$ and $\pi_{t}$ which minimize $(1 / 2)\left[\alpha\left(x_{t}-\bar{x}\right)^{2}+\left(\pi_{t}-\bar{\pi}\right)^{2}\right]+\mathcal{F}_{t}$ subject to $\pi_{t}=\lambda x_{t}+F_{t}$, where $\mathcal{F}_{t}, F_{t}$ denote remainder terms that are treated as given under discretionary policy. This leads to the first order condition

$$
\lambda\left(\pi_{t}-\bar{\pi}\right)+\alpha\left(x_{t}-\bar{x}\right)=0,
$$

so that $i_{t}$ is set to satisfy (1), (2) and (6). An explicit form will be given below.

\footnotetext{
${ }^{3}$ In general this is not an innocuous assumption, as discussed by (Leeper 1991) and (Sims 1994). See (Woodford 1996) for a full treatment of the interrelation between fiscal and monetary policy in a closely related model and (Woodford 1999) for the simplification adopted in our paper.
} 
To obtain an optimal interest rate rule under $\mathrm{RE}$ we show that there is a solution of the form

$$
\begin{gathered}
\pi_{t}=a_{1}+d_{1} u_{t} \\
x_{t}=a_{2}+d_{2} u_{t} .
\end{gathered}
$$

Under RE we have $E_{t} \pi_{t+1}=a_{1}+d_{1} \rho u_{t}, E_{t} x_{t+1}=a_{2}+d_{2} \rho u_{t}$. Inserting into (1), (2) and (6) yields

$$
\begin{gathered}
\bar{a}_{1}=\frac{\lambda(\lambda \bar{\pi}+\alpha \bar{x})}{\alpha(1-\beta)+\lambda^{2}}, \bar{d}_{1}=\frac{\alpha}{\alpha(1-\beta \rho)+\lambda^{2}} \\
\bar{a}_{2}=-\frac{\lambda \beta a_{1}-\lambda \bar{\pi}-\alpha \bar{x}}{\left(\lambda^{2}+\alpha\right)}, \bar{d}_{2}=-\frac{\lambda}{\lambda^{2}+\alpha(1-\beta \rho)} \\
i_{t}=\psi_{0}+\psi_{u} u_{t}+\psi_{g} g_{t}, \text { where } \psi_{0}=\bar{a}_{1}, \psi_{u}=\frac{(1-\rho) \lambda+\alpha \rho \varphi}{\varphi\left[\alpha(1-\beta \rho)+\lambda^{2}\right]}, \psi_{g}=\varphi^{-1} .
\end{gathered}
$$

Under this optimal policy rule the interest rate is adjusted to neutralize any shock to the IS curve. Price shocks present the policy makers with a tradeoff since they affect both inflation and output in the same direction. It can be shown that the policy makers face a trade-off between the variabilities of output and inflation and their optimal choice depends on $\alpha$ as is reflected in the coefficient $\psi_{u}$. We will call (9) the fundamentals form of the RE-optimal policy rule.

This derivation assumes both RE and that the REE takes the specified form. It is known that this interest rate rule leads to indeterminacy, i.e. a multiplicity of REE, see e.g. (Woodford 1999), Section 5.1 and (Svensson and Woodford 1999), Section 2.4. To see this point, and for our subsequent analysis of stability under learning, we require the reduced form, obtained by combining this interest rate rule (9) with (1) and (2):

$$
\left(\begin{array}{c}
\pi_{t} \\
x_{t}
\end{array}\right)=\left(\begin{array}{c}
0 \\
-\varphi \bar{a}_{1}
\end{array}\right)+\left(\begin{array}{cc}
\beta+\lambda \varphi & \lambda \\
\varphi & 1
\end{array}\right)\left(\begin{array}{c}
\hat{E}_{t} \pi_{t+1} \\
\hat{E}_{t} x_{t+1}
\end{array}\right)+\left(\begin{array}{c}
1-\lambda \varphi \psi_{u} \\
-\varphi \psi_{u}
\end{array}\right) u_{t} .
$$

It is convenient to discuss the issues of determinacy and stability under learning in a more general framework. 


\subsection{Methodology}

The reduced form (10) is a special case of the following forward-looking bivariate model

$$
\left(\begin{array}{c}
\pi_{t} \\
x_{t}
\end{array}\right)=Q+B\left(\begin{array}{c}
\hat{E}_{t} \pi_{t+1} \\
\hat{E}_{t} x_{t+1}
\end{array}\right)+C u_{t}
$$

where $B$ is $2 \times 2$ and $Q, C$ are $2 \times 1$. It is well-known that, under RE, this model is determinate if both eigenvalues of $B$ lie inside the unit circle. The unique stationary REE then gives the endogenous variables as a linear function of the exogenous shock.

We now relax the assumption that private agents have rational expectations and consider the interpretation of the temporary equilibrium in the model. If agents optimize using subjective (but possibly nonrational) probability distributions over future variables, optimal behavior is characterized by first-order necessary conditions that can be written as a sequence of Euler equations involving subjective expectations over the entire future. The Euler equation for the current period is assumed to be the behavioral rule giving the current decision as a function of the expected state next period. ${ }^{4}$ To complete the description of the agents' behavior we must supplement this Euler equation with a rule for forecasting the required state variables next period. The parameters of the forecast functions are updated using a standard adaptive learning rule such as least squares.

This interpretation means that agents behave in a boundedly rational but, in our view, reasonable way. Our approach has the advantage that it leads to a dynamical system that is formulated entirely in terms of the linearized reduced form model and standard updating equations for the parameters of the forecast functions.

Least squares and related learning dynamics have been widely studied and shown to converge to the usually employed REE in many standard models. This is true of the stationary solutions of, for example, the Cagan model of inflation, the Sargent-Wallace IS-LM-PC model, the Samuelson overlapping generations model and the real business cycle model. Recent overviews of the literature are provided e.g. in (Evans and Honkapohja 1999) and (Evans and Honkapohja 2001). In stochastic frameworks least squares and closely related learning rules are the most widely employed formulation, though

\footnotetext{
${ }^{4}$ This is appropriate only when preferences are time-separable as they are here.
} 
other learning algorithms have occasionally been considered. In this paper we follow the literature and focus on least squares learning, though we do briefly demonstrate the robustness of our results to some alternative learning schemes.

Returning to (11), the private agents are assumed to have forecast functions $^{5}$

$$
\left(\begin{array}{c}
\hat{E}_{t} \pi_{t+1} \\
\hat{E}_{t} x_{t+1}
\end{array}\right)=A_{t}+K_{t} u_{t}
$$

where the $2 \times 1$ parameter vectors

$$
A_{t}=\left(\begin{array}{l}
a_{1, t} \\
a_{2, t}
\end{array}\right) \text { and } K_{t}=\left(\begin{array}{c}
k_{1, t} \\
k_{2, t}
\end{array}\right)
$$

are updated over time according to least squares. We consider the stability under learning of some rational expectations solution $(\bar{A}, \bar{K})$, i.e. whether the estimated parameters $\left(A_{t}, K_{t}\right)$ converge to $(\bar{A}, \bar{K})$ over time. It is known for learning problems of this type that, under fairly general assumptions, convergence to REE obtains if and only if certain stability conditions, known as E-stability conditions, are satisfied. In this section we obtain the E-stability results and defer the explicit treatment of least squares learning to the next section.

The E-stability conditions are developed as follows. For given values of the parameters of the forecast functions, called the perceived law of motion, one computes the resulting actual law of motion implied by the structure. E-stability is then determined by a differential equation in notional time in which the parameters adjust in the direction of the actual law of motion parameter values. Formally, we proceed as follows.

Inserting into (11) the perceived law of motion

$$
\left(\begin{array}{c}
\hat{E}_{t} \pi_{t+1} \\
\hat{E}_{t} x_{t+1}
\end{array}\right)=A+K u_{t}, \text { where } A=\left(\begin{array}{c}
a_{1} \\
a_{2}
\end{array}\right), K=\left(\begin{array}{c}
k_{1} \\
k_{2}
\end{array}\right),
$$

gives

$$
\left(\begin{array}{l}
\pi_{t} \\
x_{t}
\end{array}\right)=Q+B A+(B K+C) u_{t}
$$

\footnotetext{
${ }^{5}$ Because the private sector is populated by a large number of "small" agents, it is natural to assume that strategic behavior in expectations formation is absent and agents simply try to learn the equilibrium processes for the endogenous variables they need to forecast.
} 
which leads to the actual law of motion, i.e. the implied forecast functions

$$
\left(\begin{array}{c}
\hat{E}_{t} \pi_{t+1} \\
\hat{E}_{t} x_{t+1}
\end{array}\right)=Q+B A+\rho(B K+C) u_{t} .
$$

We have obtained a mapping

$$
T(A, K)=(Q+B A, \rho(B K+C))
$$

from parameters of the perceived law of motion to the implied actual law of motion. An RE solution of the form (14) is a fixed point $(\bar{A}, \bar{K})$ of this mapping. E-stability of $(\bar{A}, \bar{K})$ is then defined as local asymptotic stability of the differential equation

$$
\frac{d}{d \tau}(A, K)=T(A, K)-(A, K)
$$

at $(\bar{A}, \bar{K})$, where $\tau$ denotes notional time.

For this framework E-stability conditions are readily obtained by computing the derivative of $T(A, K)-(A, K)$. For the $A$ component the condition is that the real parts of the eigenvalues $m_{1}, m_{2}$ of $B$ must be less than one. (Equivalently $|B-I|>0$ and $\operatorname{tr}(B-I)<0$ ). For the $K$ component the derivative for the map is $\rho B-I$. Thus the second set of E-stability conditions is that the real parts of $\rho m_{i}, i=1,2$, are both less than one. It follows, for the reduced form (11), that determinacy implies E-stability, but the converse does not hold. ${ }^{6}$ In particular, for the white noise case $\rho=0$ E-stability simply reduces to the first set of conditions that the eigenvalues of $B$ have real parts less than one.

While in models of the form (11) determinacy is a stricter requirement than E-stability, we caution the reader that this is not a general result. For example, consider the Muth-type model $y_{t}=\mathcal{A}+\mathcal{B} \hat{E}_{t-1} y_{t}+v_{t}$, where $v_{t}$ is a white noise vector. Provided $I-\mathcal{B}$ is nonsingular, this model has a unique stationary REE $y_{t}=(I-\mathcal{B})^{-1} \mathcal{A}+v_{t}$. The model is thus always determinate, but the REE is E-stable only if all eigenvalues of $\mathcal{B}$ have real parts less than one. In general, the relationship between determinacy and E-stability can be

\footnotetext{
${ }^{6}$ Section 2.5 gives an example of an E-stable equilibrium which is not determinate. Taylor-type interest rate rules based on forecasts of inflation and output gap can also lead to cases of indeterminacy in which the "fundamental solution" $(\bar{A}, \bar{K})$ is nevertheless E-stable, see (Bullard and Mitra 2000). Moreover, the non-fundamental REE are not E-stable, see (Honkapohja and Mitra 2001).
} 
highly complex, depending on the presence of lagged endogenous variables and the structure of the information set. This point is discussed at length in (Evans and Honkapohja 2001) for a wide variety of models.

\subsection{Instability of Fundamentals-Based Policy Rules}

We now apply this analysis to the fundamentals form of the RE optimal interest rate rule, which yielded the reduced form (10). We focus on the issue of stability under learning. Throughout this section we retain the assumption that the policy maker knows the true structure (1), (2) and the parameter values of the economy.

Before proceeding we note that if agents do not have RE then this policy rule need not satisfy the first-order condition for discretionary optimal policy, given by (6). We are therefore examining what happens out of equilibrium if policy makers follow a fundamentals based interest rate rule (9), derived under the (mistaken) assumption that the economy is in the corresponding $\mathrm{REE}$. A reasonable requirement for a good policy rule is that if private agents make small expectational errors, the economy will converge to the posited REE as agents correct these errors over time through a learning rule. As we shall see, this issue turns out to be a major concern.

Using the reduced form (10) and the general methodology of Section 2.2, we have that the derivative matrix for the vector of intercepts $A$ in (16) is

$$
B-I=\left(\begin{array}{cc}
\beta+\lambda \varphi-1 & \lambda \\
\varphi & 0
\end{array}\right) .
$$

Since $|B-I|<0$, we have E-instability. (Using the results of Section 2.2 it is also immediate that we have indeterminacy.) In the appendix we show that E-instability implies instability of the economy under least squares learning:

Proposition 1 The REE of the economy $\pi_{t}=\bar{a}_{1}+\bar{d}_{1} u_{t}, x_{t}=\bar{a}_{2}+\bar{d}_{2} u_{t}$ under the fundamentals form of the RE-optimal monetary policy rule (9) and least squares learning by private agents is unstable for all parameter values, i.e. the economy converges to the REE with probability zero.

We have stated this result in terms of least squares learning, but the instability also holds under alternative learning rules, as we will show below in Section 4.1. The intuition for the result is straightforward. If $\lambda \varphi+\beta>1$, a deviation of $\hat{E}_{t} \pi_{t+1}$ above its $\mathrm{RE}$ value leads, through the IS curve, to 
an increase in $x_{t}$ and through the Phillips curve to a higher $\pi_{t}$. Over time this leads to upward revisions of both $\hat{E}_{t} \pi_{t+1}$ and $\hat{E}_{t} x_{t+1}$. Nothing in the interest rate rule offsets this tendency, so that the economy would move cumulatively away from the REE. ${ }^{7}$ We remark that the estimated coefficients (with probability one) do not converge to any constant parameter vector, at least within the region for which the linearization is valid.

Under the fundamental based policy rule the economy will follow unstable trajectories with agents making systematic errors in forecasting despite their efforts to improve their forecast functions. Moreover, policy makers will find that their first-order condition for discretionary optimum is persistently failing to hold. Presumably, both agents and policy makers will change their behavior in the face of these divergent paths. We do not pursue this issue further here. Our point is simply that the policy (9), which is consistent with discretionary optimal policy under RE, fails to be robust to small initial deviations from RE.

In fact, Proposition 1 is a special case of the next proposition which states that all interest rate rules that depend linearly only on the fundamentals are unstable:

Proposition 2 Consider a policy rule of the form $i_{t}=\eta_{0}+\eta_{u} u_{t}+\eta_{g} g_{t}$ and an associated REE of the form $\pi_{t}=a_{1}+d_{1} u_{t}+n_{1} g_{t}, x_{t}=a_{2}+d_{2} u_{t}+n_{2} g_{t}$. For all values of the structural parameters, the REE is unstable under least squares learning by private agents.

The proof of this result follows from the observation that the coefficient matrix of the expectations is the same as in the reduced form (10) for optimal policy (the coefficients of the policy rule only affect the constant and disturbance terms). E-stability depends only on this matrix. A predecessor of this result is (Howitt 1992) who showed instability under learning of interest rate pegging in several monetary models.

This strong instability result raises the question of whether there are alternative monetary policy rules that are robust to least squares learning and implement the optimal policy without commitment.

\subsection{An Expectations Based Optimal Rule}

The instability problem of the preceding section resulted from the implicit assumption by the policy maker that private agents have perfectly rational

\footnotetext{
${ }^{7}$ Using (10), a more refined intuition can be developed for the case $\lambda \varphi+\beta<1$.
} 
expectations at every point in time. If there is a chance that they are not fully rational it would seem natural instead to base policy in part directly on expectations of private agents. In this section we assume that these expectations are observable and consider a policy rule that includes a dependence on them. ${ }^{8}$

The relevant policy rule is obtained by solving for $i_{t}$ from the structural equations (1), (2) and the optimality condition (6). ${ }^{9}$ We obtain

$$
i_{t}=\delta_{0}+\delta_{\pi} \hat{E}_{t} \pi_{t+1}+\delta_{x} \hat{E}_{t} x_{t+1}+\delta_{g} g_{t}+\delta_{u} u_{t}
$$

where the coefficients are

$$
\begin{aligned}
& \delta_{0}=-\left(\lambda^{2}+\alpha\right)^{-1} \varphi^{-1}(\lambda \bar{\pi}+\alpha \bar{x}), \\
& \delta_{\pi}=1+\left(\lambda^{2}+\alpha\right)^{-1} \varphi^{-1} \lambda \beta \\
& \delta_{x}=\varphi^{-1} \\
& \delta_{g}=\varphi^{-1} \\
& \delta_{u}=\left(\lambda^{2}+\alpha\right)^{-1} \varphi^{-1} \lambda .
\end{aligned}
$$

By construction, the rule (17) implements optimal discretionary policy in every period and for all values of private expectations. It thus achieves this policy even out of equilibrium. We will refer to (17) as the expectations based optimal rule.

The reduced form is

$$
\begin{aligned}
\left(\begin{array}{c}
\pi_{t} \\
x_{t}
\end{array}\right)= & \left(\begin{array}{cc}
\beta \alpha\left(\lambda^{2}+\alpha\right)^{-1} & 0 \\
-\beta \lambda\left(\lambda^{2}+\alpha\right)^{-1} & 0
\end{array}\right)\left(\begin{array}{c}
\hat{E}_{t} \pi_{t+1} \\
\hat{E}_{t} x_{t+1}
\end{array}\right) \\
& +\left(\begin{array}{c}
-\varphi \delta_{0} \lambda \\
-\varphi \delta_{0}
\end{array}\right)+\left(\begin{array}{c}
1-\varphi \delta_{u} \lambda \\
-\varphi \delta_{u}
\end{array}\right) u_{t} .
\end{aligned}
$$

Since the eigenvalues of the $B$ matrix are 0 and $0<\beta \alpha\left(\lambda^{2}+\alpha\right)^{-1}<1$, we have determinacy and, therefore, also E-stability under this interest rate rule. In the appendix we show that this implies convergence under least squares learning:

\footnotetext{
${ }^{8}$ Alternatively, if the policy maker knows the learning rules of private agents, it can infer the expectations from other observed data.

${ }^{9}$ We assume that the policy maker does not make active use of the learning behavior on the part of agents. Strategic manipulation by the policy maker of agents' learning rules merits investigation in future research.
} 
Proposition 3 For all parameter values the REE of the economy under the expectations based optimal rule is determinate and is stable under least squares learning by private agents, i.e. the economy converges almost surely to the REE that corresponds to the optimal monetary policy without commitment. ${ }^{10}$

A partial intuition for this result is that, for example, an increase in $\hat{E}_{t} \pi_{t+1}$ leads to an increase in $i_{t}$ which more than offsets the direct effect of $\hat{E}_{t} \pi_{t+1}$ on $x_{t}$ since $\delta_{\pi}>1$. In economic terms the real interest rate is increased which reduces the output gap. Thus our policy rule satisfies the "Taylor principle." Likewise, the expectations based rule tightens policy in the face of higher $\hat{E}_{t} x_{t+1}$. The rule (17) with the specified parameter values succeeds in guiding the expectations of private agents and the economy to the optimal REE.

We have stressed that (17) implements the first-order condition (6) for all possible values of private expectations. One might ask if there are other interest rate rules with this property. Consider interest rate rules of the form

$$
i_{t}=\omega_{0}+\zeta_{x} x_{t}+\zeta_{\pi} \pi_{t}+\omega_{x} \hat{E}_{t} x_{t+1}+\omega_{\pi} \hat{E}_{t} \pi_{t+1}+\omega_{g} g_{t}+\omega_{u} u_{t}
$$

Clearly, a continuum of rules of this form exist which satisfy the first-order condition (6). For example, set $\omega_{i}=\delta_{i}, i=x, \pi, g, u, \omega_{0}=\delta_{0}+\zeta_{0}$ with $\zeta_{i}$, $i=x, \pi, 0$ chosen to ensure that (6) is met. In particular, one can choose any $\zeta_{i}$ so that $\zeta_{0}+\zeta_{x} x_{t}+\zeta_{\pi} \pi_{t}$ is a multiple of $\alpha\left(x_{t}-\bar{x}\right)+\lambda\left(\pi_{t}-\bar{\pi}\right)$. (Obviously, such rules would have to examined for determinacy and E-stability.) In comparison to (17), these rules have the disadvantage that they require additional information, namely current output and inflation data. ${ }^{11}$

One might also ask whether one could set $\omega_{x}=\omega_{\pi}=0$ and still implement (6). The following argument shows that this is not possible. Note first that (2) and (6) determine $x_{t}$ and $\pi_{t}$ uniquely as linear functions of $\hat{E}_{t} \pi_{t+1}$ and $u_{t}$. Substituting these and (19) with $\omega_{x}=\omega_{\pi}=0$ into (1) it is seen that the resulting equation cannot hold for all possible expectations and exogenous shocks. Thus any rule that implements (6) for all values of expectations must

\footnotetext{
${ }^{10}$ In fact, formally the convergence of learning is even global, i.e. it obtains for all initial parameter estimates. Since the economic model is based on a local linearization we do not emphasize global stability in the proposition.

${ }^{11}$ (McCallum 1999), p.1517, argues that policies depending on current $x_{t}$ and $\pi_{t}$ are not operational, because it is unrealistic to assume that policy makers have this information.
} 
depend directly on those expectations. Our rule (17) is the simplest rule of this class, since it does not depend on $x_{t}$ or $\pi_{t}$.

We remark that the determinacy result under the rule (17) appears to contrast sharply with the findings of (Bernanke and Woodford 1997) who also considered policies that depend on private expectations. The key to our determinacy and stability results is that the policy rule (17) uses the economic structure and all available information. (Svensson and Woodford 1999), section 4.2, also show that certain "hybrid" interest rate rules that depend on private expectations can achieve determinacy.

Of course, formulating the optimal expectations based rule requires knowledge of the structural parameters. There are two points to be made. First, if the policy makers follow the rule (17) with parameters deviating from the specified values by small amounts, the economy will converge over time to an REE that deviates from the optimum by small amounts. Second, the policy maker could try to learn the true values of the structural parameters. This raises the issue of whether simultaneous learning by private agents and the policy maker can still lead the economy to converge to the optimal REE. We take this up in Section 3. Before doing so, we briefly consider an alternative interest rate rule that aims to implement optimal discretionary policy.

\subsection{An Alternative Expectations Based Rule}

Under RE the optimal monetary policy without commitment can be characterized in various ways as emphasized by (Woodford 1999). In particular, (Clarida, Gali, and Gertler 1999) point out that the optimal interest rate can be written as

$$
i_{t}=-\frac{(1-\rho) \lambda}{\rho \alpha \varphi} \bar{a}_{1}+\left(1+\frac{(1-\rho) \lambda}{\rho \alpha \varphi}\right) E_{t} \pi_{t+1}+\varphi^{-1} g_{t} .
$$

This form suggests an alternative expectations based policy rule in which rational expectations $E_{t} \pi_{t+1}$ is replaced by observed private expectations $\hat{E}_{t} \pi_{t+1}$. We note that such a rule makes use of some rational expectations assumptions to eliminate expectations of the output gap. It is of interest to know the properties of this rule. 
The reduced form is now

$$
\begin{aligned}
\left(\begin{array}{c}
\pi_{t} \\
x_{t}
\end{array}\right)= & \left(\begin{array}{cc}
\beta-(1-\rho) \lambda^{2} / \rho \alpha & \lambda \\
-(1-\rho) \lambda / \rho \alpha & 1
\end{array}\right)\left(\begin{array}{c}
\hat{E}_{t} \pi_{t+1} \\
\hat{E}_{t} x_{t+1}
\end{array}\right) \\
& +\left(\begin{array}{c}
-\bar{a}_{1}(1-\rho) \lambda^{2} / \rho \alpha \\
-\bar{a}_{1}(1-\rho) \lambda / \rho \alpha
\end{array}\right)+\left(\begin{array}{c}
1 \\
0
\end{array}\right) u_{t} .
\end{aligned}
$$

It can be verified that the REE is E-stable, so that optimal discretionary policy would also be obtained asymptotically under this expectations based rule. This stability result shows the importance of strong positive feedback from private inflation expectations to interest rates.

Despite the stability result this alternative rule is less appealing than our proposed rule (17). First, the optimal REE under the alternative rule is not necessarily determinate. Indeterminacy invariably arises for values of $\rho$ close to zero. This reflects the very large reaction of the interest rate to inflation expectations, see (20). Second, the large coefficient for small $|\rho|$ is troubling for another reason: if $\rho$ is not known exactly and replaced by an estimate, then small estimation errors cause large deviations from optimal REE. Finally, in contrast to (17) the policy rule (20) does not implement the optimal discretionary policy out of equilibrium.

\section{Learning by the Policy Maker}

We now consider an extension of the analysis in which the key structural parameter values are unknown. The policy maker is assumed to know the structure of the economy but must estimate the parameter values using observed data. ${ }^{12}$ In order to make the estimation problem more realistic we introduce unobserved shocks to the model (1), (2).

The IS and Phillips curves thus take the form

$$
\begin{gathered}
x_{t}=-\varphi\left(i_{t}-\hat{E}_{t} \pi_{t+1}\right)+\hat{E}_{t} x_{t+1}+g_{t}+e_{x, t} \\
\pi_{t}=\lambda x_{t}+\beta \hat{E}_{t} \pi_{t+1}+u_{t}+e_{\pi, t},
\end{gathered}
$$

where now $x_{t}, \pi_{t}, e_{x, t}$ and $e_{\pi, t}$ are not observable at time $t . g_{t}, u_{t}$ are observable at $t$ and $x_{t}, \pi_{t}$ are observed with a lag. $e_{x, t}, e_{\pi, t}$ are independent white

\footnotetext{
${ }^{12}$ Thus in contrast to (Sargent 1999) our policy maker estimates a correctly specified structural model.
} 
noise and $g_{t}, u_{t}, e_{x, t}, e_{\pi, t}$ are exogenous and mutually independent. The private expectations are assumed to be observable. We also assume that the discount factor $\beta$, a parameter shared by private agents and the policy maker, is known. $\alpha, \bar{\pi}, \bar{x}$ in the objective function are, of course, taken to be known. However, the key structural parameters $\varphi$ and $\lambda$ must be estimated.

To complete the description of the extended model we must specify the behavior of the policy maker. The objective function (5) remains as before, but with our current informational assumptions the first order condition becomes

$$
\lambda\left(E_{t} \pi_{t}-\bar{\pi}\right)+\alpha\left(E_{t} x_{t}-\bar{x}\right)=0
$$

It can be verified that with the unobserved shocks the RE solution takes the form

$$
\begin{aligned}
& \pi_{t}=\bar{a}_{1}+\bar{d}_{1} u_{t}+\lambda e_{x, t}+e_{\pi, t} \\
& x_{t}=\bar{a}_{2}+\bar{d}_{2} u_{t}+e_{x, t},
\end{aligned}
$$

where the parameter values are given in (7) and (8). The fundamentals form of the RE-optimal rule remains unchanged, i.e. it takes the form (9) and the expectations based rule continues to be of the form (17).

We now turn to the formulation of learning and provide explicit equations for the updating rules. The private agents' forecast functions have the same form as before (12), where the parameters are estimated by least squares. At any given time, the agents run regressions using the available data and therefore they update their parameter estimates each period as new data become available. In line with the literature on least squares learning we give the equations in recursive form. ${ }^{13}$

Let

$$
\xi_{1, t}=\left(\begin{array}{c}
a_{1, t} \\
k_{1, t}
\end{array}\right), \xi_{2, t}=\left(\begin{array}{c}
a_{2, t} \\
k_{2, t}
\end{array}\right), U_{t}=\left(\begin{array}{c}
1 \\
u_{t}
\end{array}\right)
$$

so that the forecasts can be written as $\hat{E}_{t} \pi_{t+1}=\xi_{1, t}^{\prime} U_{t}$ and $\hat{E}_{t} x_{t+1}=\xi_{2, t}^{\prime} U_{t}$. Recall that $a_{i, t}, k_{i, t}, i=1,2$, are the parameters of the forecast functions

\footnotetext{
${ }^{13}$ The general approach used here was introduced by (Marcet and Sargent 1989). See (Evans and Honkapohja 1999) for a recent survey and (Evans and Honkapohja 2001) for a detailed treatment.
} 
defined in (13). Under recursive least squares (RLS) learning the parameter vectors are updated according to the formulae

$$
\begin{aligned}
\xi_{1, t} & =\xi_{1, t-1}+t^{-1} R_{U, t-1}^{-1} U_{t-2}\left(\pi_{t-1}-\xi_{1, t-1}^{\prime} U_{t-2}\right) \\
\xi_{2, t} & =\xi_{2, t-1}+t^{-1} R_{U, t-1}^{-1} U_{t-2}\left(x_{t-1}-\xi_{2, t-1}^{\prime} U_{t-2}\right) \\
R_{U, t} & =R_{U, t-1}+t^{-1}\left(U_{t-1} U_{t-1}^{\prime}-R_{U, t-1}\right) .
\end{aligned}
$$

This recursion requires starting values for the parameters at time $t=0$ and it is well-known that for appropriate starting values RLS is equivalent to the standard least squares formula. In the formulation above the parameter estimates at time $t$ use data through period $t-1 .^{14}$ The time $t$ forecasts do, however, use the current observation of the exogenous variable $u_{t}$. Note that the procedure used by private agents estimates the parameters of the reduced form, which is all that is required for forecasting purposes.

The policy maker uses RLS to estimate the structural parameters $\varphi, \lambda$ and $\rho$, which are required for setting optimal monetary policy. Let

$$
\begin{aligned}
y_{1, t} & =x_{t}-\hat{E}_{t} x_{t+1}-g_{t} \\
y_{2, t} & =\pi_{t}-\beta \hat{E}_{t} \pi_{t+1}-u_{t} \\
r_{t} & =i_{t}-\hat{E}_{t} \pi_{t+1} .
\end{aligned}
$$

Because $r_{t}$ depends directly only on the variables $g_{t}$ and $u_{t}$, and $x_{t}$ depends only on $g_{t}, u_{t}$ and $e_{x, t}$, consistent estimates of $\varphi$ and $\lambda$ can be obtained by regressions of $y_{1, t}$ on $r_{t}$ and $y_{2, t}$ on $x_{t}$, respectively. ${ }^{15}$ Thus the policy maker uses least squares to estimate

$$
\begin{aligned}
& y_{1, t}=-\varphi\left(i_{t}-\hat{E}_{t} \pi_{t+1}\right)+e_{x, t} \text { and } \\
& y_{2, t}=\lambda x_{t}+e_{\pi, t} .
\end{aligned}
$$

If required, a consistent estimate of $\rho$ can of course be obtained by a regression of $u_{t}$ on $u_{t-1} \cdot{ }^{16}$

\footnotetext{
${ }^{14}$ This is common practice in the literature. Including current data would create a simultaneity complication that is convenient to avoid and would not alter the central results.

${ }^{15}$ If $e_{x, t}$ and $e_{\pi, t}$ were correlated then an instrumental variable estimator would be needed to obtain a consistent estimator of $\lambda$.

${ }^{16}$ An estimate of $\rho$ is needed for the RE-optimal policy but not for the expectations based optimal policy.
} 
In recursive form this becomes ${ }^{17}$

$$
\begin{aligned}
\hat{\varphi}_{t} & =\hat{\varphi}_{t-1}+t^{-1} R_{r, t-1}^{-1} r_{t-1}\left(y_{1, t-1}+\hat{\varphi}_{t-1} r_{t-1}\right) \\
R_{r, t} & =R_{r, t-1}+t^{-1}\left(r_{t-1}^{2}-R_{r, t-1}\right) \\
\hat{\lambda}_{t} & =\hat{\lambda}_{t-1}+t^{-1} R_{x, t-1}^{-1} x_{t-1}\left(y_{2, t-1}-\hat{\lambda}_{t-1} x_{t-1}\right) \\
R_{x, t} & =R_{x, t-1}+t^{-1}\left(x_{t-1}^{2}-R_{x, t-1}\right) \\
\hat{\rho}_{t} & =\hat{\rho}_{t-1}+t^{-1} R_{u, t-1}^{-1} u_{t-1}\left(u_{t}-\hat{\rho}_{t-1} u_{t-1}\right) \\
R_{u, t} & =R_{u, t-1}+t^{-1}\left(u_{t}^{2}-R_{u, t-1}\right) .
\end{aligned}
$$

It remains to specify the monetary policy rules. The fundamentals form of the RE-optimal rule based on estimated parameters takes the form

$$
\begin{aligned}
i_{t} & =\hat{a}_{1, t}+\hat{\psi}_{t} u_{t}+\hat{\varphi}_{t}^{-1} g_{t} \\
\text { where } \hat{a}_{1, t} & =\frac{\hat{\lambda}_{t}\left(\hat{\lambda}_{t} \bar{\pi}+\alpha \bar{x}\right)}{\alpha(1-\beta)+\hat{\lambda}_{t}^{2}} \text { and } \hat{\psi}_{t}=\frac{\left(1-\hat{\rho}_{t}\right) \hat{\lambda}_{t}+\alpha \hat{\rho}_{t} \hat{\varphi}_{t}}{\hat{\varphi}_{t}\left[\alpha\left(1-\hat{\rho}_{t} \beta\right)+\hat{\lambda}_{t}^{2}\right]} .
\end{aligned}
$$

The expectations based optimal policy rule with estimated structural parameters is

$$
i_{t}=\hat{\delta}_{0, t}+\hat{\delta}_{\pi, t} \hat{E}_{t} \pi_{t+1}+\hat{\delta}_{x, t} \hat{E}_{t} x_{t+1}+\hat{\delta}_{g, t} g_{t}+\hat{\delta}_{u, t} u_{t}
$$

where the coefficients are

$$
\begin{aligned}
& \hat{\delta}_{0, t}=-\left(\hat{\lambda}_{t}^{2}+\alpha\right)^{-1} \hat{\varphi}_{t}^{-1}\left(\hat{\lambda}_{t} \bar{\pi}+\alpha \bar{x}\right) \\
& \hat{\delta}_{\pi, t}=1+\left(\hat{\lambda}_{t}^{2}+\alpha\right)^{-1} \hat{\varphi}_{t}^{-1} \hat{\lambda}_{t} \beta \\
& \hat{\delta}_{x, t}=\hat{\varphi}_{t}^{-1} \\
& \hat{\delta}_{g, t}=\hat{\varphi}_{t}^{-1} \\
& \hat{\delta}_{u, t}=\left(\hat{\lambda}_{t}^{2}+\alpha\right)^{-1} \hat{\varphi}_{t}^{-1} \hat{\lambda}_{t} .
\end{aligned}
$$

Given parameter estimates and forecasts, the temporary equilibrium is determined by (21), (22) and the policy rule (24) or (25), respectively.

In the appendix we demonstrate the following result:

\footnotetext{
${ }^{17}$ The formulations for $\hat{\varphi}_{t}$ and $\hat{\lambda}_{t}$ vary slightly from least squares since we have introduced an additional lag in the equations for $R_{r, t}$ and $R_{x, t}$. This is convenient for expressing the system in standard form as a stochastic recursive algorithm. Note that the regression of $y_{1, t}$ on $r_{t}$ gives an estimate of $-\varphi$. This explains the plus in front of $\hat{\varphi}_{t-1}$ in the first recursion below.
} 
Proposition 4 Suppose that both the policy maker and the private agents are learning with parameter estimates updated according to the RLS algorithms specified above. Then

(i) If policy makers follow the fundamentals form of the RE-optimal rule (24), the REE is unstable for all parameter values.

(ii) If policy makers follow the expectations based optimal rule (25) then, for all parameter values, the estimates $\left(\hat{\varphi}_{t}, \hat{\lambda}_{t}\right)$ converge locally to $(\varphi, \lambda)$, the expectations of private agents converge locally to RE values, and the economy converges locally to the REE that corresponds to the optimal monetary policy without commitment.

The notion of local convergence has several precise interpretations, as discussed in the appendix. In particular convergence is not guaranteed unless the policy maker has some a priori information concerning the possible values of the structural parameters. However, Proposition 4 shows that the fundamental contrast between the instability result in part (i) and the stability result in part (ii) continues to hold when the policy maker is learning the structure of the economy.

Our results illustrate the potential feasibility of optimal monetary policy when private expectations are observable and the rule is formulated to react to these expectations. Even when the structural parameters are unknown to policy makers and agents are not endowed with rational expectations, the economy is (locally) stable when both parties follow natural procedures to estimate the key parameters.

\section{Extensions}

In this section we take up several extensions. First we show that our central results extend to variations in the learning rules used by private agents. Next we examine the robustness of our results to observation errors. Finally, we consider a number of variations to the model or the formulation of monetary policy. For simplicity, in this section we assume the policy maker knows the true parameter values.

\subsection{Alternative Learning Rules}

Although the literature on adaptive learning in stochastic models has focused on least squares learning, a number of alternatives have also been considered. 
We here show that our key results are not limited to the least squares framework. ${ }^{18}$

We first consider learning based on SG (stochastic gradient) algorithms. These have been proposed by, among others, (Sargent 1993) and (Kuan and White 1994). This algorithm attempts to minimize the expected sum of squared forecast errors. In the current context, private agents would update parameters according to

$$
\begin{aligned}
& \xi_{1, t}=\xi_{1, t-1}+t^{-1} U_{t-2}\left(\pi_{t-1}-\xi_{1, t-1}^{\prime} U_{t-2}\right) \\
& \xi_{2, t}=\xi_{2, t-1}+t^{-1} U_{t-2}\left(x_{t-1}-\xi_{2, t-1}^{\prime} U_{t-2}\right) .
\end{aligned}
$$

This algorithm is simpler than RLS in that these updating equations are not dependent on the matrix of second moments of the regressor $U_{t}$. For regression set-ups with fixed parameters and standard assumptions, the SG algorithm will provide consistent estimates of the parameters, though it does not possess the optimality properties of least squares. It does, however, have the advantage of being simpler and faster to compute than least squares.

The analysis in (Evans and Honkapohja 1998) can be applied here. It is shown there that the convergence conditions for SG learning for a class of models is given by the E-stability conditions that also govern convergence of least squares learning. (The framework of (Evans and Honkapohja 1998) has expectations of current rather than future variables. However, because here we consider RE solutions of an analogous form, the same argument can be applied.) It follows that both our instability and stability results carry over to SG learning.

As a second example, we consider a simple misspecified learning rule. Suppose that private agents ignore the dependence of $\pi_{t}$ and $x_{t}$ on $u_{t}$ and forecast each variable using simple averages. Formulating this recursively we have $\hat{E}_{t} \pi_{t+1}=a_{1, t}$ and $\hat{E}_{t} x_{t+1}=a_{2, t}$ where

$$
\begin{aligned}
& a_{1, t}=a_{1, t-1}+t^{-1}\left(\pi_{t-1}-a_{1, t-1}\right), \\
& a_{2, t}=a_{2, t-1}+t^{-1}\left(x_{t-1}-a_{2, t-1}\right) .
\end{aligned}
$$

As in Section 3 this can be set up as a stochastic recursive algorithm. It is straightforward to show that the system remains unstable under the fundamentals based monetary policy rule and that the economy is stable under

\footnotetext{
${ }^{18}$ It can be shown that the stability results in Propositions 3 and 4 also hold for other stability criteria under learning that have been proposed in the literature. For example, the REE under the expectations based policy rule is both strongly and iteratively E-stable, see e.g. (Evans and Honkapohja 2001), Chapters 9 and 15 for these concepts.
} 
the expectations based policy rule. In the latter case private expectations $\left(a_{1, t}, a_{2, t}\right)$ will converge to $\left(\bar{a}_{1}, \bar{a}_{2}\right)$ and the economy converges to a "restricted perceptions equilibrium." 19 In this equilibrium private sector expectations are equal to the unconditional mathematical expectations of $\pi_{t}$ and $x_{t}$. However, they are not fully rational (when $\rho \neq 0$ ) since they ignore the dependence on $u_{t}$ and are therefore not equal to the conditional mathematical expectations. Nevertheless, the expectations based policy rule continues to provide the optimal monetary policy without commitment, given the way agents form their expectations.

\subsection{Observation Errors}

We next consider the issue of observation errors, starting with the case in which the exogenous variables $g_{t}, u_{t}$ are subject to measurement error. We use the set-up (21)-(22), but assume that policy makers know the values of the structural parameter. For simplicity we restrict attention to the case where $g_{t}$ and $u_{t}$ are serially uncorrelated. Suppose that the policy maker observes $\tilde{g}_{t}$ and $\tilde{u}_{t}$, where

$$
\begin{aligned}
\tilde{g}_{t} & =g_{t}+\varepsilon_{t}, \text { where } \varepsilon_{t} \sim \operatorname{iid}\left(0, \sigma_{\varepsilon}^{2}\right) \\
\tilde{u}_{t} & =u_{t}+v_{t}, \text { where } v_{t} \sim i i d\left(0, \sigma_{v}^{2}\right) .
\end{aligned}
$$

Provided the variances are known, the policy maker faces a signal extraction problem with a straightforward solution. The linear projection of $g_{t}$ on $\tilde{g}_{t}$ is given by

$$
P\left[g_{t} \mid \tilde{g}_{t}\right]=\zeta_{g} \tilde{g}_{t}, \text { where } \zeta_{g}=\frac{\sigma_{g}^{2}}{\sigma_{g}^{2}+\sigma_{\varepsilon}^{2}} .
$$

Analogously, the linear projection of $u_{t}$ is

$$
P\left[u_{t} \mid \tilde{u}_{t}\right]=\zeta_{u} \tilde{u}_{t}, \text { where } \zeta_{u}=\frac{\sigma_{u}^{2}}{\sigma_{u}^{2}+\sigma_{v}^{2}} .
$$

The first order condition for optimal discretionary policy is again given by (23). Under optimal policy the REE takes the form

$$
\begin{aligned}
& \pi_{t}=a_{1}+b_{1} g_{t}+c_{1} \varepsilon_{t}+d_{1} u_{t}+f_{1} v_{t}+\lambda e_{x, t}+e_{\pi, t} \\
& x_{t}=a_{2}+b_{2} g_{t}+c_{2} \varepsilon_{t}+d_{2} u_{t}+f_{2} v_{t}+e_{x, t},
\end{aligned}
$$

\footnotetext{
${ }^{19}$ This is the terminology we use in (Evans and Honkapohja 2001).
} 
where

$$
\begin{aligned}
a_{1} & =\bar{a}_{1}, a_{2}=\bar{a}_{2} \\
b_{1} & =\lambda\left(1-\zeta_{g}\right), b_{2}=1-\zeta_{g} \\
c_{1} & =-\lambda \zeta_{g}, c_{2}=-\zeta_{g} \\
d_{1} & =1-\left(\alpha+\lambda^{2}\right)^{-1} \lambda^{2} \zeta_{u}, d_{2}=-\left(\alpha+\lambda^{2}\right)^{-1} \lambda \zeta_{u} \\
f_{1} & =d_{1}-1, f_{2}=d_{2} .
\end{aligned}
$$

We note that, under optimal discretionary policy, $x_{t}$ and $\pi_{t}$ now respond to $g_{t}$ as well as $u_{t}$ shocks because of the measurement error. The analysis of learning is, however, virtually unchanged and the key results carry through as before: the economy is unstable under the RE optimal policy rule and stable under the expectations based optimal rule. ${ }^{20}$

Another potentially important complication arises in connection with the expectations based rule if private sector expectations are measured with error. Clearly the optimality properties of our rule would be undermined if these errors are sizeable, but we can show that our policy rule is robust to small measurement errors in private expectations. Thus suppose that the expectations based policy rule (17) is modified to be

$$
i_{t}=\delta_{0}+\delta_{\pi}\left(\hat{E}_{t} \pi_{t+1}+w_{\pi, t}\right)+\delta_{x}\left(\hat{E}_{t} x_{t+1}+w_{x, t}\right)+\delta_{g} g_{t}+\delta_{u} u_{t},
$$

where $w_{\pi, t}$ and $w_{x, t}$ denote white noise measurement errors with finite variances. The parameter values of the policy rule are unchanged. For private agents we continue to assume perceived laws of motion of the form (14). For the basic model (1)-(2), this policy rule leads to the reduced form (18) augmented by additive linear terms in $w_{x, t}$ and $w_{\pi, t}$. The mapping from the perceived to the actual law of motion is thus the same as for Section 2.4. Therefore this expectations based policy rule remains stable, with private agents converging to rational expectations. This result holds even if the observation errors $w_{x, t}$ and $w_{\pi, t}$ are large, though with large observation errors the resulting equilibrium would be far from the discretionary optimum. However, it is easily seen that if $w_{x, t}$ and $w_{\pi, t}$ have small variances then the economy under the expectations based rule is close to the discretionary optimum.

The preceding paragraph assumed that the policy makers know the true structural parameters. If instead they estimate structural parameters using

\footnotetext{
${ }^{20}$ In this set-up private sector forecasts depend on $g_{t}$ as well as $u_{t}$.
} 
RLS as in Section 3 there is the additional complication that, while $\hat{\lambda}_{t}$ remains consistent, the estimator $\hat{\varphi}_{t}$ becomes inconsistent due to measurement error in the regressor $r_{t}$. However, if $w_{\pi, t}$ has a small variance then the inconsistency will be small and the equilibrium will be approximately optimal asymptotically. Alternatively, policy makers could obtain a consistent estimate of $\varphi$ using recursive instrumental variables with lagged measured $r_{t}$ as instrument.

In summary, although observation errors of the fundamental shocks or private expectations introduce complications for our proposed policy rule, we have seen that these difficulties can be largely overcome. The case of substantial measurement errors in private expectations is sufficiently important to warrant a separate treatment.

\subsection{Other Directions}

\subsubsection{Output Inertia}

A number of variations of the new Phillips curve model have been taken up in the policy literature, including endogenous inflation and output inertia. We consider briefly the more straightforward case of output persistence due to costs of adjustment. As shown in (Clarida, Gali, and Gertler 1999), Section 6 , with output persistence the IS curve becomes

$$
x_{t}=-\varphi\left(i_{t}-\hat{E}_{t} \pi_{t+1}\right)+\theta x_{t-1}+(1-\theta) \hat{E}_{t} x_{t+1}+g_{t},
$$

where $0<\theta<1$. The corresponding expectations based policy rule is then

$$
i_{t}=\delta_{0}+\delta_{\pi} \hat{E}_{t} \pi_{t+1}+\delta_{x}(1-\theta) \hat{E}_{t} x_{t+1}+\varphi^{-1} \theta x_{t-1}+\delta_{g} g_{t}+\delta_{u} u_{t},
$$

where the parameters $\delta_{i}$ for $i=0, \pi, x, g, u$ are the same as in (17). We restrict attention to the case $\rho=0$, as do (Clarida, Gali, and Gertler 1999). In this case the private sector forecasts under rational expectations are appropriate constants.

Under our rule (27) the reduced form is

$$
\begin{aligned}
& x_{t}=\left(\lambda^{2}+\alpha\right)^{-1}(\lambda \bar{\pi}+\alpha \bar{x})-\frac{\lambda \beta}{\alpha+\lambda^{2}} \hat{E}_{t} \pi_{t+1} \\
& \pi_{t}=\left(\lambda^{2}+\alpha\right)^{-1} \lambda(\lambda \bar{\pi}+\alpha \bar{x})+\frac{\beta \alpha}{\alpha+\lambda^{2}} \hat{E}_{t} \pi_{t+1}+u_{t} .
\end{aligned}
$$


Assuming now that agents form their forecast $\hat{E}_{t} \pi_{t+1}$ as the average of past inflation rates, it is easily verified that forecasts converge to the REE corresponding to the optimal policy without commitment. Thus output persistence presents no difficulties. The case of inflation persistence is more complicated and is therefore omitted.

\subsubsection{Policy Under Limited Commitment}

Our discussion of optimal policies has been under the assumption that the policy maker does not have commitment power. The ability to commit to a given rule raises several additional issues, as discussed Section 4 of (Clarida, Gali, and Gertler 1999). For brevity we assume here that $\bar{x}=0=\bar{\pi}$, so that the classic inflationary bias issue does not arise. Nevertheless, commitment can still result in gains over the discretionary equilibrium.

(Clarida, Gali, and Gertler 1999), Section 4.2.1 argue that a simple family of rules yielding an REE of the form

$$
x_{t}=\omega u_{t}
$$

can dominate the optimum without commitment. (Note that with $\bar{x}=0=\bar{\pi}$ the optimal REE without commitment also has this form.) Suppose that policy makers have limited commitment power in that they are able only to commit to policies that take this form. The optimal policy with commitment in this class is formally identical to the model without commitment when the relative weight on output $\alpha$ is replaced by a smaller value $\alpha^{c}=\alpha(1-\beta \rho)$. It is thus apparent that our instability and stability results can be applied for this family of rules with limited commitment.

Optimal policy under full commitment power does not generally have this form, as emphasized by (Woodford 1999). The general analysis of issues raised in this paper for monetary policy with full commitment will require a separate study.

\subsubsection{Rules Based on Inflation and Output Data}

A natural question to ask is whether it is possible to devise monetary policy rules that are based on inflation and output data, rather than on observed private expectations, and that are stable under learning and lead to the optimal policy without commitment. That is, can one side-step the apparent 
need to observe private expectations in order to implement the optimal discretionary policy rule? There are two separate issues, which we take up in turn.

First, is it possible using observed current data to infer the values of private expectations and insert them into (17) to obtain an optimal discretionary policy for all values of private expectations? The answer to this is clearly negative since in Section 2.4 we showed that implementing optimal discretionary policy requires the interest rate to depend on private expectations and $u_{t}, g_{t}$ even if it also depends on $x_{t}, \pi_{t}$. The source of the difficulty arises from the simultaneity implied by using observations on these variables to deduce private expectations while setting $i_{t}$ as a function of these private expectations. Further practical difficulties with inferring expectations from other data are that the values of structural parameters may be unknown and that there may be unobserved shocks as in (21)-(22).

We do not wish to overstate this particular argument. With additional auxiliary assumptions it may be possible to deduce the values of private expectations from the history of data. For example, if it were known that all agents form expectations by following the least squares learning rules that we have analyzed, then it would be straightforward to use this information to infer $\hat{E}_{t} \pi_{t+1}$ and $\hat{E}_{t} x_{t+1}$ and implement (17). Such a policy would be entirely in the spirit of this paper. But whether such an inference is possible is a delicate matter that depends on details of the model, the information structure and auxiliary assumptions. It would appear preferable to obtain direct observations on expectations.

Another question is whether judicious use of the rational expectations assumption can be used to substitute observations of actual inflation and output gap for expected inflation and expected output gap in the optimal interest rate rule. Such a rule would not implement the optimal discretionary policy outside equilibrium, but the economy under the rule might possibly converge to the REE with optimal discretionary policy.

We consider two possibilities within the context of the basic model of Section 2.1. Under rational expectations and optimal policy we have $\pi_{t}=$ $\bar{a}_{1}+\bar{d}_{1} u_{t}$ and $x_{t}=\bar{a}_{2}+\bar{d}_{2} u_{t}$. From $E_{t} \pi_{t+1}=\bar{a}_{1}+\bar{d}_{1} \rho u_{t}$ and $E_{t} x_{t+1}=$ $\bar{a}_{2}+\bar{d}_{2} \rho u_{t}$. it follows that $E_{t} \pi_{t+1}=\bar{a}_{1}+\rho\left(\pi_{t}-\bar{a}_{1}\right)$ and $E_{t} x_{t+1}=\bar{a}_{2}+\rho\left(x_{t}-\bar{a}_{2}\right)$. Using these expressions to replace $\hat{E}_{t} \pi_{t+1}$ and $\hat{E}_{t} x_{t+1}$ in the expectations based rule (17) suggests the policy

$$
i_{t}=\delta_{0}^{\prime}+\delta_{\pi} \rho \pi_{t}+\delta_{x} \rho x_{t}+\delta_{g} g_{t}+\delta_{u} u_{t},
$$


where the values of $\delta_{i}, i=\pi, x, g, u$ are as in equation (17) and $\delta_{0}^{\prime}$ is an appropriate constant. Alternatively one could start with the fundamentals based rule (9) and use the relationship $u_{t}=\bar{d}_{1}^{-1}\left(\pi_{t}-\bar{a}_{1}\right)$ to obtain the policy rule

$$
i_{t}=\psi_{0}^{\prime}+\left(\varphi^{-1} \alpha^{-1} \lambda(1-\rho)+\rho\right) \pi_{t}+\varphi^{-1} g_{t},
$$

where $\psi_{0}^{\prime}$ is an appropriate constant.

Will these rules be stable under least-squares learning by private agents? It is easily established that stability is not guaranteed. For the case $\rho=0$ the rule (28) reduces to $i_{t}=\delta_{0}^{\prime}+\delta_{g} g_{t}+\delta_{u} u_{t}$. But we have already established in Section 2.3 that any policy that depends linearly only on the fundamental shocks cannot be E-stable and hence is unstable under least squares learning. Using continuity of eigenvalues, it follows that the instability result holds for all nonzero $|\rho|$ sufficiently small. A similar argument applies to the rule (29) for $\lambda / \alpha$ and $|\rho|$ sufficiently small.

The rules (28) and (29) are particular cases of rules based on actual inflation and output gap data. There are a number of simple interest rate rules along such lines that do not explicitly aim for optimal policy. (Bullard and Mitra 2000) consider various general classes of such rules and derive conditions on policy rule parameter values that yield stability under learning. Another class of rules is based on controlling the money stock, and one could consider the stability under learning of the REE for monetary feedback rules. See Part III of (Evans and Honkapohja 2001) for examples of the latter. These examples do not exhaust the list of policy rules that have been proposed in the recent literature. We emphasize that it is important to analyze, for any proposed policy rule, whether the RE equilibrium of interest is robust to expectational errors, i.e. is stable under learning.

\section{Conclusions}

The central message of the paper is both simple and fundamental. First, optimal monetary policy should not assume perfectly rational expectations on the part of private agents: Even if the initial deviations from perfect rationality are small, the economy will diverge when the fundamentals form of the RE-optimal policy rule is followed.

Second, the instability problem can be overcome if the set of conditioning variables is augmented to include observed private expectations. We have 
shown how to use the economic structure to devise an expectations based optimal policy rule. Under this interest rate rule policy makers implement optimal discretionary policy in each period and the economy converges over time to the corresponding REE.

Our stability results hold even when the policy maker is learning the required values of the structural parameters at the same time that private agents are following adaptive learning rules. The propositions have been demonstrated both under the standard assumption that private expectations are revised in accordance with least squares learning and under some other natural learning algorithms.

The analysis has been conducted under the assumption that accurate data on private expectations are available. This allows us to present sharp and powerful results. Appropriately conditioning policy on private expectations gives due weight to the role of these expectations in determining the dynamics of the economy, which the assumption of perfect RE suppresses. Obtaining accurate data on private expectations should therefore be a high priority for monetary policy makers. Utilizing the appropriate form of dependence of policy on private expectations is also crucial, since not all rules that depend on private forecasts lead to stability under learning.

\section{Appendix}

In this appendix we give the proof of Proposition 4 and comment on the modifications for the proofs for Propositions 1 and 3. Consider the case of the expectations based policy rule (25). Combining this equation with the IS curve (21) and the private agent forecast rules (12) we obtain the law of motion for $x_{t}$ under learning:

$$
\begin{aligned}
x_{t}= & -\varphi \hat{\varphi}_{t}^{-1}\left(a_{2, t}+k_{2, t} u_{t}\right)-\varphi \hat{\varphi}_{t}^{-1} g_{t} \\
& -\varphi \hat{\varphi}_{t}^{-1}\left(\hat{\lambda}_{t}^{2}+\alpha\right)^{-1}\left(\hat{\lambda}_{t} \beta\left(a_{1, t}+k_{1, t} u_{t}\right)-\hat{\lambda}_{t}\left(u_{t}+\bar{\pi}\right)-\alpha \bar{x}\right) \\
& +a_{2, t}+k_{2, t} u_{t}+g_{t}+e_{x, t},
\end{aligned}
$$

which is of the form

$$
x_{t}=x\left(\hat{\varphi}_{t}, \hat{\lambda}_{t}, \xi_{1, t}, \xi_{2, t} ; u_{t}, g_{t}, e_{x, t}, e_{\pi, t}\right) .
$$

Similarly, using also the PC curve (22) and the definitions of $r_{t}, y_{1, t}$ and $y_{2, t}$ 
we can obtain

$$
\begin{aligned}
\pi_{t} & =\pi\left(\hat{\varphi}_{t}, \hat{\lambda}_{t}, \xi_{1, t}, \xi_{2, t} ; u_{t}, g_{t}, e_{x, t}, e_{\pi, t}\right) \\
r_{t} & =r\left(\hat{\varphi}_{t}, \hat{\lambda}_{t}, \xi_{1, t}, \xi_{2, t} ; u_{t}, g_{t}, e_{x, t}, e_{\pi, t}\right) \\
y_{1, t} & =y_{1}\left(\hat{\varphi}_{t}, \hat{\lambda}_{t}, \xi_{1, t}, \xi_{2, t} ; u_{t}, g_{t}, e_{x, t}, e_{\pi, t}\right) \\
y_{2, t} & =y_{2}\left(\hat{\varphi}_{t}, \hat{\lambda}_{t}, \xi_{1, t}, \xi_{2, t} ; u_{t}, g_{t}, e_{x, t}, e_{\pi, t}\right) .
\end{aligned}
$$

Define the parameter vector

$$
\theta_{t}^{\prime}=\left(\hat{\varphi}_{t}, \hat{\lambda}_{t}, \xi_{1, t}^{\prime}, \xi_{2, t}^{\prime}, R_{x, t}, R_{\pi, t}, \operatorname{vec}\left(R_{U, t}\right)\right)
$$

and the state vector

$$
X_{t}=\left(u_{t}, u_{t-1}, u_{t-2}, g_{t-1}, e_{x, t-1}, e_{\pi, t-1}\right) .
$$

The recursive equations for the parameter estimates can then be written in the form

$$
\theta_{t}=\theta_{t-1}+t^{-1} \mathcal{H}\left(\theta_{t-1}, X_{t}\right)
$$

so that theorems on the convergence of stochastic recursive algorithms can be applied, see e.g. part II of (Evans and Honkapohja 2001). These results state that, under appropriate regularity conditions, convergence of these algorithms is governed by the stability of the associated ordinary differential equation (ODE)

$$
\frac{d \theta}{d \tau}=h(\theta) \text { where } h(\theta)=\lim _{t \rightarrow \infty} \operatorname{EH}\left(\theta, X_{t}\right) .
$$

For the case of the expectations based optimal policy one can compute for the policy maker's parameter estimates

$$
\begin{aligned}
\frac{d \hat{\varphi}}{d \tau} & =R_{r}^{-1} E r_{t-1}(\theta)^{2}(\varphi-\hat{\varphi}) \\
\frac{d R_{r}}{d \tau} & =E r_{t-1}(\theta)^{2}-R_{r} \\
\frac{d \hat{\lambda}}{d \tau} & =R_{x}^{-1} E x_{t-1}(\theta)^{2}(\lambda-\hat{\lambda}) \\
\frac{d R_{x}}{d \tau} & =E x_{t-1}(\theta)^{2}-R_{x}
\end{aligned}
$$


where

$$
r_{t}(\theta)=r\left(\hat{\varphi}, \hat{\lambda}, \xi_{1}, \xi_{2}, \hat{\rho} ; u_{t}, g_{t}, e_{x, t}, e_{\pi, t}\right)
$$

and

$$
x_{t}(\theta)=x\left(\hat{\varphi}, \hat{\lambda}, \xi_{1}, \xi_{2}, \hat{\rho} ; u_{t}, g_{t}, e_{x, t}, e_{\pi, t}\right)
$$

For the private agents' parameter estimates we get

$$
\begin{aligned}
& \frac{d \xi_{1}}{d \tau}=R_{U}^{-1}\left(E U_{t-2} U_{t-2}^{\prime}\right) \lambda \\
& {\left[-\varphi \hat{\varphi}^{-1}\left(\begin{array}{c}
a_{2} \\
k_{2} \rho
\end{array}\right)-\frac{\varphi \hat{\varphi}^{-1} \hat{\lambda}}{\hat{\lambda}^{2}+\alpha}\left(\begin{array}{c}
\beta a_{1}-\bar{\pi}-\hat{\lambda}^{-1} \alpha \bar{x} \\
\left(\beta k_{1}+1\right) \rho
\end{array}\right)+\left(\begin{array}{c}
a_{2} \\
k_{2} \rho
\end{array}\right)\right]} \\
& +R_{U}^{-1}\left(E U_{t-2} U_{t-2}^{\prime}\right)\left[\left(\begin{array}{c}
\beta a_{1} \\
\left(\beta k_{1}+1\right) \rho
\end{array}\right)-\left(\begin{array}{c}
a_{1} \\
k_{1}
\end{array}\right)\right] \\
& \frac{d \xi_{2}}{d \tau}=R_{U}^{-1}\left(E U_{t-2} U_{t-2}^{\prime}\right) \\
& {\left[-\varphi \hat{\varphi}^{-1}\left(\begin{array}{c}
a_{2} \\
k_{2} \rho
\end{array}\right)-\frac{\varphi \hat{\varphi}^{-1} \hat{\lambda}}{\hat{\lambda}^{2}+\alpha}\left(\begin{array}{c}
\beta a_{1}-\bar{\pi}-\hat{\lambda}^{-1} \alpha \bar{x} \\
\left(\beta k_{1}+1\right) \rho
\end{array}\right)+\left(\begin{array}{c}
0 \\
k_{2}(\rho-1)
\end{array}\right)\right]} \\
& \frac{d R_{U}}{d \tau}=E U_{t-2} U_{t-2}^{\prime}-R_{U}
\end{aligned}
$$

It can be verified that this system has a unique equilibrium point $\theta^{*}$ at which

$$
\begin{aligned}
\hat{\varphi} & =\varphi, \hat{\lambda}=\lambda, \xi_{1}=\bar{\xi}_{1} \equiv\left(\bar{a}_{1}, \bar{k}_{1}\right)^{\prime}, \xi_{2}=\bar{\xi}_{2} \equiv\left(\bar{a}_{2}, \bar{k}_{2}\right)^{\prime} \\
R_{r} & =E r_{t-1}\left(\theta^{*}\right)^{2}, R_{x}=E x_{t-1}\left(\theta^{*}\right)^{2}, R_{U}=E U_{t-2} U_{t-2}^{\prime}
\end{aligned}
$$

It can be shown that local stability is governed by the following "small" ODE

$$
\begin{aligned}
& \frac{d \hat{\varphi}}{d \tau}=\varphi-\hat{\varphi} \\
& \frac{d \hat{\lambda}}{d \tau}=\lambda-\hat{\lambda}
\end{aligned}
$$




$$
\begin{aligned}
& \frac{d \xi_{1}}{d \tau}= \lambda\left[-\varphi \hat{\varphi}^{-1}\left(\begin{array}{c}
a_{2} \\
k_{2} \rho
\end{array}\right)-\frac{\varphi \hat{\varphi}^{-1} \hat{\lambda}}{\hat{\lambda}^{2}+\alpha}\left(\begin{array}{c}
\beta a_{1}-\bar{\pi}-\hat{\lambda}^{-1} \alpha \bar{x} \\
\left(\beta k_{1}+1\right) \rho
\end{array}\right)+\left(\begin{array}{c}
a_{2} \\
k_{2} \rho
\end{array}\right)\right] \\
&+ {\left[\left(\begin{array}{c}
\beta a_{1} \\
\left(\beta k_{1}+1\right) \rho
\end{array}\right)-\left(\begin{array}{c}
a_{1} \\
k_{1}
\end{array}\right)\right] } \\
& \frac{d \xi_{2}}{d \tau}=-\varphi \hat{\varphi}^{-1}\left(\begin{array}{c}
a_{2} \\
k_{2} \rho
\end{array}\right)-\frac{\varphi \hat{\varphi}^{-1} \hat{\lambda}}{\hat{\lambda}^{2}+\alpha}\left(\begin{array}{c}
\beta a_{1}-\bar{\pi}-\hat{\lambda}^{-1} \alpha \bar{x} \\
\left(\beta k_{1}+1\right) \rho
\end{array}\right)+\left(\begin{array}{c}
0 \\
k_{2}(\rho-1)
\end{array}\right) .
\end{aligned}
$$

Clearly the subsystem in $(\hat{\varphi}, \hat{\lambda})$ is stable with $\hat{\varphi} \rightarrow \varphi, \hat{\lambda} \rightarrow \lambda$. Hence local stability of the REE is determined by the ODE

$$
\begin{gathered}
\frac{d \xi_{1}}{d \tau}=-\frac{\lambda^{2}}{\lambda^{2}+\alpha}\left(\begin{array}{c}
\beta a_{1}-\bar{\pi}-\lambda^{-1} \alpha \bar{x} \\
\left(\beta k_{1}+1\right) \rho
\end{array}\right)+\left(\begin{array}{c}
\beta a_{1} \\
\left(\beta k_{1}+1\right) \rho
\end{array}\right)-\left(\begin{array}{c}
a_{1} \\
k_{1}
\end{array}\right) \\
\frac{d \xi_{2}}{d \tau}=-\frac{\lambda}{\lambda^{2}+\alpha}\left(\begin{array}{c}
\beta a_{1}-\bar{\pi}-\lambda^{-1} \alpha \bar{x} \\
\left(\beta k_{1}+1\right) \rho
\end{array}\right)-\left(\begin{array}{c}
a_{2} \\
k_{2}
\end{array}\right) .
\end{gathered}
$$

These equations can be restacked as a system that is identical to the general E-stability equation (16) when

$$
T(A, K)=\left(\begin{array}{cc}
\frac{\lambda}{\lambda^{2}+\alpha}(\lambda \bar{\pi}+\alpha \bar{x})+\frac{\alpha}{\lambda^{2}+\alpha} \beta a_{1} & \rho \frac{\alpha}{\lambda^{2}+\alpha}\left(1+\beta k_{1}\right) \\
\frac{1}{\lambda^{2}+\alpha}\left(\lambda \bar{\pi}+\alpha \bar{x}-\lambda \beta a_{1}\right) & -\rho \frac{\lambda}{\lambda^{2}+\alpha}\left(1+\beta k_{1}\right)
\end{array}\right),
$$

which is the $T$-map (15) corresponding to the reduced form (18) in Section 2.4 , and which was shown to be asymptotically stable.

The standard results from the learning literature can be now applied. The regularity conditions on the function $\mathcal{H}\left(\theta, X_{t}\right)$, and bounded moment conditions for the exogenous innovations, required for the local convergence results in Chapter 6 of (Evans and Honkapohja 2001), are easily seen to be satisfied. Above we saw that the equilibrium of the large ODE $\theta^{*}$ is locally asymptotically stable. Hence the basic local convergence theorems apply. There are various senses of local probabilistic convergence. For example, almost sure convergence obtains if the algorithm is augmented to have a projection facility constraining estimates to a neighborhood of the REE. For details see Chapter 6 of (Evans and Honkapohja 2001) or (Evans and Honkapohja 1999). This proves part (ii) of Proposition 4. 
The proof of part (i) of Proposition 4 follows a similar sequence of steps, but with the alternative policy rule to obtain law of motion for $x_{t}, \pi_{t}, r_{t}, y_{1, t}$ and $y_{2, t}$. For this rule the parameter vector $\theta_{t}$ must be augmented to include $\hat{\rho}_{t}$ and $R_{u, t}$. The stability properties of the associated ODE can again be shown to depend on the stability properties of the E-stability differential equation. The latter is locally unstable, as shown in Section 2.3, and the nonconvergence results found in Chapter 6 of (Evans and Honkapohja 2001) or (Evans and Honkapohja 1999) can be applied. These results state that $\theta_{t}$ converges to $\theta^{*}$ (or to any other point) with probability zero.

Turning to Propositions 1 and 3 the parameter vector $\theta_{t}$ is now

$$
\theta_{t}^{\prime}=\left(\xi_{1, t}^{\prime}, \xi_{2, t}^{\prime}, \operatorname{vec}\left(R_{U, t}\right)\right)
$$

For the expectation based rule the associated ODE is

$$
\begin{aligned}
& \frac{d \xi_{1}}{d \tau}=R_{U}^{-1}\left(E U_{t-2} U_{t-2}^{\prime}\right) \\
& {\left[-\frac{\lambda^{2}}{\lambda^{2}+\alpha}\left(\begin{array}{c}
\beta a_{1}-\bar{\pi}-\lambda^{-1} \alpha \bar{x} \\
\left(\beta k_{1}+1\right) \rho
\end{array}\right)+\left(\begin{array}{c}
\beta a_{1} \\
\left(\beta k_{1}+1\right) \rho
\end{array}\right)-\left(\begin{array}{c}
a_{1} \\
k_{1}
\end{array}\right)\right]} \\
& \frac{d \xi_{2}}{d \tau}=R_{U}^{-1}\left(E U_{t-2} U_{t-2}^{\prime}\right)\left[-\frac{\lambda}{\lambda^{2}+\alpha}\left(\begin{array}{c}
\beta a_{1}-\bar{\pi}-\lambda^{-1} \alpha \bar{x} \\
\left(\beta k_{1}+1\right) \rho
\end{array}\right)-\left(\begin{array}{c}
a_{2} \\
k_{2}
\end{array}\right)\right] \\
& \frac{d R_{U}}{d \tau}=E U_{t-2} U_{t-2}^{\prime}-R_{U}
\end{aligned}
$$

It can be seen that for this system the equilibrium point

$$
\left(\theta^{*}\right)^{\prime}=\left(\bar{\xi}_{1}^{\prime}, \bar{\xi}_{2}^{\prime}, \operatorname{vec}\left(E U_{t-2} U_{t-2}^{\prime}\right)\right)
$$

is globally asymptotically stable. It can be verified that the assumptions are satisfied for the global stability result in Chapter 6 of (Evans and Honkapohja 2001), which should be consulted for technical details. It follows that $\theta_{t} \rightarrow \theta^{*}$ with probability one, proving Proposition 3. For the fundamentals form of the RE-optimal rule we obtain a differential equation system in $\left(\frac{d \xi_{1}}{d \tau}, \frac{d \xi_{2}}{d \tau}, \frac{d R_{U}}{d \tau}\right)$, which is locally unstable at $\theta^{*}$. Thus $\theta_{t} \rightarrow \theta^{*}$ with probability zero, establishing Proposition 1. 


\section{References}

BAnk Of England, U. K. (2001): Inflation Report. Bank of England, www.bankofengland.co.uk/inflationreport/index.htm.

Bernanke, B., And M. Woodford (1997): "Inflation Forecasts and Monetary Policy," Working paper, NBER WP6157.

Blinder, A. S. (1998): Central Banking in Theory and Practice. MIT Press, Cambridge, Mass.

Bullard, J., and K. Mitra (2000): "Learning About Monetary Policy Rules," mimeo.

Clarida, R., J. Gali, and M. Gertler (1999): "The Science of Monetary Policy: A New Keynesian Perspective," Journal of Economic Literature, 37, 1661-1707.

Evans, G. W., and S. Honkapohja (1998): "Stochastic Gradient Learning in the Cobweb Model," Economic Letters, 61, 333-337.

chap. 7 , pp. 449-542.

(2001): Learning and Expectations in Macroeconomics. Princeton University Press, Princeton, New Jersey.

Federal Reserve Bank, K. C. (1984): Price Stability and Public Policy. Federal Reserve Bank of Kansas City, Kansas City.

HALL, R. E. (1984): "Monetary Strategy with an Elastic Price Standard," in (Federal Reserve Bank 1984), chap. 6, pp. 137-159.

Honkapohja, S., and K. Mitra (2001): "Are Non-Fundamental Equilibria Learnable in Models of Monetary Policy?," Working paper, University of Helsinki.

HowitT, P. (1992): "Interest Rate Control and Nonconvergence to Rational Expectations," Journal of Political Economy, 100, 776-800.

Kuan, C.-M., and H. White (1994): "Adaptive Learning with Nonlinear Dynamics Driven by Dependent Processes," Econometrica, 62, 1087-1114. 
Leeper, E. M. (1991): “Equilibria under 'Active' and 'Passive' monetary and Fiscal Policies," Journal of Monetary Economics, 27, 129-147.

Marcet, A., and T. J. Sargent (1989): "Convergence of Least-Squares Learning Mechanisms in Self-Referential Linear Stochastic Models," Journal of Economic Theory, 48, 337-368.

McCallum, B. T. (1999): "Issues in the Design of Monetary Policy Rules," in (Taylor and Woodford 1999), chap. 23, pp. 1483-1530.

Sargent, T. J. (1993): Bounded Rationality in Macroeconomics. Oxford University Press, Oxford.

(1999): The Conquest of American Inflation. Princeton University Press, Princeton NJ.

Sims, C. A. (1994): "A Simple Model for Study of the Determination of the Price Level and the Interaction of Monetary and Fiscal Policy," Economic Theory, 4, 381-399.

Svensson, L. E. O., and M. Woodford (1999): "Implementing Optimal Policy through Inflation-Forecast Targeting," Working paper.

Taylor, J. (1993): "Discretion versus Policy Rules in Practice," CarnegieRochester Conference Series in Public Policy, 39, 195-214.

Taylor, J., And M. Woodford (eds.) (1999): Handbook of Macroeconomics, Volume 1. Elsevier, Amsterdam.

Woodford, M. (1996): "Control of the Public Debt: A Requirement for Price Stability?," Working paper, NBER WP5684.

(1999): "Optimal Monetary Policy Inertia," Working paper. 